
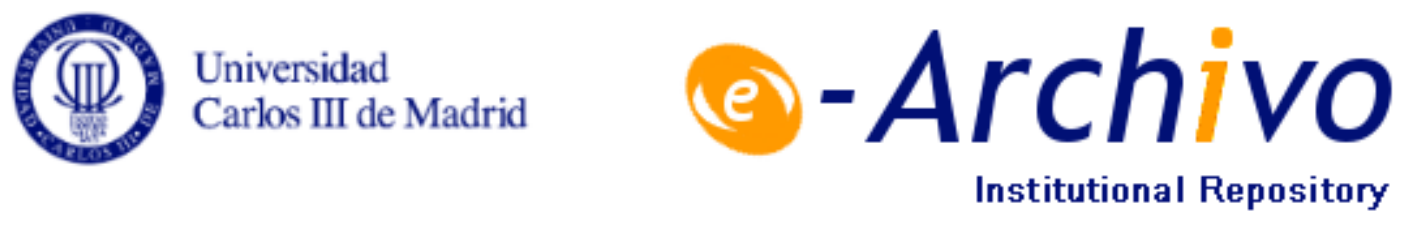

This is a postprint version of the following published document:

Proceedings of Institution of Mechanical Engineers, Part C: Journal of Mechanical Engineering Science, vol. 226, issue C12 (2012) pp. 2912-2924

DOI http://dx.doi.org/10.1177/0954406212442289

(C) 2012 Sage Publications 


\title{
On the use of variable-separation method for the analysis of vibration problems with time-dependent boundary conditions
}

\author{
Josué Aranda-Ruiz and José Fernández-Sáez
}

\begin{abstract}
In this article, the axial vibrations of a rod with a clamped end and the transversal vibrations of a cantilever beam, both with a time-dependent and non-harmonic force applied on their free ends, are analysed. These are problems in which the traction and the shear, for the rod and the beam, respectively, prescribed in the boundaries of the bodies vary with time. The problems can be solved by the method proposed by Mindlin and Goodman. However, it is usual to solve this problem by the classic variable-separation method (which does not properly fulfil the time-dependent boundary conditions). The displacements and the forces along the systems are derived from both cited methods, and the results are compared. These results highlight the importance of using the proper solution method for the vibration problems with time-dependent boundary conditions.
\end{abstract}

\section{Keywords}

Axial vibrations, transversal vibrations, time-dependent boundary conditions, natural frequencies, variable-separation method

\section{Introduction}

Topics on mechanical vibrations of continuum systems, such as rods, beams, plates and shells, constitute a critical subject for engineers and scientists working in civil, mechanical and aerospace fields. Thus, there are many textbooks which deal with the analysis of these subjects. $^{1-4}$

In many situations these problems can be formulated in terms or linear partial differential equations which can be solved by the classical variable-separation method, but in some cases, the displacements or tractions prescribed in the boundary of the body vary with time. This is the case of the axially vibrating cantilever beam with a time-dependent force $P(t)$ acting on its free end in the axial direction or the same beam subjected to an arbitrary transverse time-dependent displacement at its free end. In such cases, in general, the method of the separation of variables breaks down when applied directly because it is not possible to satisfy the nonharmonic time-dependent boundary conditions.

This kind of problems can be solved by the method of Laplace transform or, alternatively, by other approaches such as the procedures proposed by
Mindlin and Goodman, ${ }^{5}$ and Courant and Hilbert, ${ }^{6}$ which requires less sophisticated mathematical techniques.

However, some texts ${ }^{1}$ present examples of vibration problems with arbitrary time-dependent boundary conditions solved by the classical method of separation of variables.

In this article, the axial vibrations of a rod with a clamped end and the transversal vibrations of a cantilever beam, both with a time-dependent and nonharmonic force $P(t)$ applied on their free ends, are analysed. In the following section 'Problem formulation' is done. The solutions are reached, in the 'Solution with time-dependent boundary conditions' section, using the method proposed by Mindlin and Goodman $^{5}$ and the results are compared with those

Department of Continuum Mechanics and Structural Analysis, University Carlos III of Madrid, Leganés, Spain

\section{Corresponding author:}

Josué Aranda-Ruiz, Department of Continuum Mechanics and Structural Analysis, University Carlos III of Madrid, Avda. de la Universidad 30, 289II Leganés, Madrid, Spain.

Email: jaranda@ing.uc3m.es 
calculated, in the 'Solution with homogenous boundary conditions' section, by the classical variable-separation method (which does not properly fulfil the time-dependent boundary conditions). Then 'Comparison of results with practical examples' is made, points out important differences in the axial force (for the rod problem), shear force (for the beam problem) and displacement distributions.

\section{Problem formulation}

The problems to be studied are the axial vibrations of a clamped rod and the transverse vibrations of a cantilever beam, both with a time-dependent and non-harmonic force $P(t)$ applied on their free ends.

There are two ways to approach the solution of both problems. The first is to consider a forced-vibration problem, applying the point load as an external load applied on each body, and the boundary conditions as homogeneous conditions. ${ }^{1}$ This method does not rigorously fulfil the boundary conditions, because of the stresses at the free end of each body are considered null. The second method is to address a free-vibration problem, considering the point load applied as a boundary condition at the free end of each body, ${ }^{2}$ this method being completely rigorous with the boundary conditions of the problem.

\section{Clamped rod problem}

We consider the axial vibrations of a uniform rod of density $\rho$, Young's modulus $E$, length $L$ and crosssectional area $A$. The axial vibrations of the rod under the action of an axially distributed force $\bar{F}(\bar{x}, \bar{t})$ are governed by ${ }^{4}$

$$
\rho A \frac{\partial^{2} \bar{u}}{\partial \bar{t}^{2}}-\frac{\partial}{\partial \bar{x}}\left(E A \frac{\partial \bar{u}}{\partial \bar{x}}\right)=\bar{F}(\bar{x}, \bar{t})
$$

where $\bar{u}(\bar{x}, \bar{t})$ represents the longitudinal displacement.

With the use of new dimensionless variables defined as

$$
x=\frac{\bar{x}}{L}, \quad t=\frac{c_{r}}{L} \bar{t}, \quad u(x, t)=\frac{\bar{u}(x, t)}{L}
$$

where

$$
c_{r}=\sqrt{\frac{E}{\rho}}
$$

is a material-dependent constant called wave velocity. Equation (1) can be rewritten, considering a rod with uniform parameters, as

$$
\frac{\partial^{2} u}{\partial t^{2}}-\frac{\partial^{2} u}{\partial x^{2}}=F(x, t)
$$

where

$$
F(x, t)=\frac{\bar{F}(x, t) L}{E A}
$$

is a dimensionless axially distributed force.

The problem to be addressed is that of a clamped rod under the action of a time-dependent point load, at its free end, as shown in Figure 1.

It is easy to see that this load exerts an axial force along the rod, including its free end.

\section{Cantilever beam problem}

In this case, we consider the transversal vibrations $\bar{v}(\bar{x}, \bar{t})$ of a uniform beam, which are governed by

$$
\rho A \frac{\partial^{2} \bar{v}}{\partial \bar{t}^{2}}+\frac{\partial^{2}}{\partial \bar{x}^{2}}\left(E I \frac{\partial^{2} \bar{v}}{\partial \bar{x}^{2}}\right)=\bar{F}(\bar{x}, \bar{t})
$$

Equation (6) can be rewritten in a dimensionless form as

$$
\frac{\partial^{2} v}{\partial t^{2}}+\frac{\partial^{4} v}{\partial x^{4}}=F(x, t)
$$

with the use of new dimensionless variables defined as

$$
t=\frac{c_{b}}{L^{2}} \bar{t}, \quad F(x, t)=\frac{\bar{F}(x, t) L^{3}}{E I}, \quad v(x, t)=\frac{\bar{v}(x, t)}{L}
$$

where

$$
c_{b}=\sqrt{\frac{E I}{\rho A}}
$$

The problem to be solved is that of a cantilever beam with a time-dependent point load at its free end, as shown in Figure 2.

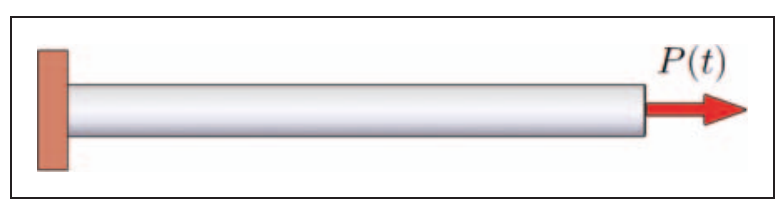

Figure I. Clamped rod with a time-dependent load applied on its free end.

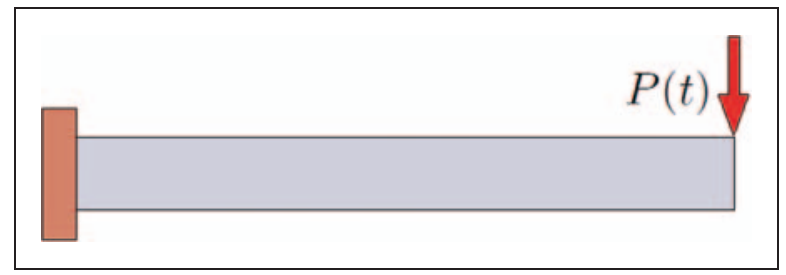

Figure 2. Cantilever beam with a time-dependent load applied on its free end. 


\section{Solution with homogeneous boundary conditions}

In this section, the classic variable-separation method to solve the two problems introduced before is used. First, the clamped rod problem is resolved and then the cantilever beam one is treated.

\section{Clamped rod problem}

As discussed, in this case, the point load is considered to be an external load, which is dependent only on time, applied at the free end of the rod. Being a point load and not a distributed load, the motion equation given by equation (4) should be rewritten as

$$
\frac{\partial^{2} u}{\partial t^{2}}-\frac{\partial^{2} u}{\partial x^{2}}=P(t) \delta(x-1)
$$

Boundary conditions for this problem are homogeneous

$$
u(0, t)=0, \quad u^{\prime}(1, t)=0
$$

as they are considered zero displacement in the clamped end, and zero axial force at the free end.

Both the initial displacement and initial velocity will be considered null, so that the initial conditions of the problem are

$$
u(x, 0)=0, \quad \dot{u}(x, 0)=0
$$

Equations (10) to (12) define a classical problem of forced vibrations, with homogeneous boundary conditions, the solution of which is achieved by the method of separation of variables. ${ }^{4}$ Therefore

$$
u(x, t)=\sum_{j=1}^{\infty} \phi_{j}(x) \eta_{j}(t)
$$

where

$$
\phi_{j}(x)=\sin \left(\omega_{j} x\right)
$$

and

$$
\eta_{j}(t)=\frac{2 \phi_{j}(1)}{\omega_{j}} \int_{0}^{t} P(\tau) \sin \left(\omega_{j}(t-\tau)\right) \mathrm{d} \tau
$$

with

$$
\omega_{j}=\frac{(2 j-1) \pi}{2}
$$

Equations (16) and (14) define the dimensionless natural frequencies and the eigenmodes of the rod, respectively. The dimensional natural frequencies are given by

$$
\bar{\omega}_{j}=\frac{c_{r}}{L} \omega_{j}
$$

The axial force along the rod is determined by ${ }^{4}$

$$
N(x, t)=\frac{\partial u}{\partial x}(x, t)=\frac{\bar{N}(x, t)}{E A}
$$

\section{Cantilever beam problem}

For the problem shown in Figure 2, the motion equation given by equation (7) can be rewritten as

$$
\frac{\partial^{2} v}{\partial t^{2}}+\frac{\partial^{4} v}{\partial x^{4}}=P(t) \delta(x-1)
$$

Homogeneous boundary conditions for the cantilever beam are

$$
\begin{aligned}
& v(0, t)=0, \quad v^{\prime}(0, t)=0 \\
& v^{\prime \prime}(1, t)=0, \quad v^{\prime \prime \prime}(1, t)=0
\end{aligned}
$$

which imply null displacement and rotation at the fixed end and null bending moment and shear force at the free end, respectively.

The same initial conditions as in previous problem will be considered, i.e.

$$
v(x, 0)=0, \quad \dot{v}(x, 0)=0
$$

The solution of the problem defined by Equations (19), (21), and 20(a) and (b), is given again by the method of separation of variables. Therefore

$$
v(x, t)=\sum_{j=1}^{\infty} \phi_{j}(x) \eta_{j}(t)
$$

where, in this problem

$$
\begin{aligned}
\phi_{j}(x)= & \sin \left(\sqrt{\omega_{j}} x\right)-\sinh \left(\sqrt{\omega_{j}} x\right) \\
& +D_{j}\left[\cos \left(\sqrt{\omega_{j}} x\right)-\cosh \left(\sqrt{\omega_{j}} x\right)\right]
\end{aligned}
$$

and

$$
\eta_{j}(t)=\frac{\phi_{j}(1)}{\omega_{j} \int_{0}^{1} \phi_{j}^{2}(x) \mathrm{d} x} \int_{0}^{t} P(\tau) \sin \left(\omega_{j}(t-\tau)\right) \mathrm{d} \tau
$$

with

$$
D_{j}=\frac{\cos \left(\sqrt{\omega_{j}}\right)+\cosh \left(\sqrt{\omega_{j}}\right)}{\sin \left(\sqrt{\omega_{j}}\right)-\sinh \left(\sqrt{\omega_{j}}\right)}
$$


In this case, the dimensionless natural frequencies of vibration are the solutions of the transcendental equation given by

$$
\cos \left(\sqrt{\omega_{j}}\right) \cosh \left(\sqrt{\omega_{j}}\right)=-1
$$

where

$$
\begin{aligned}
& \omega_{1}=3.5156, \quad \omega_{2}=22.0336, \quad \omega_{3}=61.7010 \quad \\
& \omega_{4}=120.9120, \quad \omega_{5}=199.8548, \quad \omega_{6}=298.5638
\end{aligned}
$$

For the beam problem, these dimensionless frequencies are related to the dimensional ones by

$$
\bar{\omega}_{j}=\frac{c_{b}}{L^{2}} \omega_{j}
$$

The bending moment and shear force along the beam are determined, respectively, by

$$
\begin{aligned}
& M(x, t)=\frac{\partial^{2} v}{\partial x^{2}}(x, t)=\frac{\bar{M}(x, t) L}{E I} \\
& V(x, t)=\frac{\partial^{3} v}{\partial x^{3}}(x, t)=\frac{\bar{V}(x, t) L^{2}}{E I}
\end{aligned}
$$

\section{Solution with time-dependent boundary conditions}

Again, it will now solve the case of a clamped rod subjected to a point load at its free end, $\bar{P}(\bar{t})$. This case will be considered as a free-vibrations problem, since the load will not be considered as an externally applied load, but as a boundary condition at the free end.

The partial differential equation governing the longitudinal vibrations of the rod for a free-vibrations problem is derived from equation (4), by imposing $F(x, t)=0$, as

$$
\frac{\partial^{2} u}{\partial t^{2}}-\frac{\partial^{2} u}{\partial x^{2}}=0
$$

Boundary and initials conditions are given, respectively, by

$$
u(0, t)=0, \quad u^{\prime}(1, t)=H P(t)
$$

and

$$
u(x, 0)=0, \quad \dot{u}(x, 0)=0
$$

where

$$
H=\frac{P_{0}}{E A}
$$

is a dimensionless group, and $P(t)$ is given by

$$
P(t)=\frac{\bar{P}(t)}{P_{0}}
$$

In addressing the cantilever beam problem as a problem of free vibrations, equation (7) turns into

$$
\frac{\partial^{2} v}{\partial t^{2}}+\frac{\partial^{4} v}{\partial x^{4}}=0
$$

In this case, the initial conditions are again given by equation (21), and boundary conditions are now

$$
\begin{aligned}
& v(0, t)=0, \quad v^{\prime}(0, t)=0 \\
& v^{\prime \prime}(1, t)=0, \quad v^{\prime \prime \prime}(1, t)=K P(t)
\end{aligned}
$$

where

$$
K=\frac{P_{0} L^{2}}{E I}
$$

is another dimensionless group, and $P(t)$ is given by equation (34).

\section{General solution method}

As can be seen in equations (31) and (36(b)), the boundary conditions for both problems are time dependent. In cases like this, in general, the classical method of the separation of variables breaks down when applied directly because it is not possible to satisfy the nonharmonic time-dependent boundary conditions.

In this section, a description is given, in a general way, of the method of solving problems in which the boundary conditions are functions of time, and then the resolution of the specific cases under study is continued.

The method described below also applies even taking into account damping, but this has been omitted for simplicity.

In the following discussion, the symbol $D^{(n)}$ is used to represent a linear differential operator of order $n$, given by

$$
D^{(n)}=L^{1-n} \frac{\partial^{n}}{\partial x^{n}}
$$

where $L$ is the length of the rod. This operator can be used to define the boundary conditions of the problem, so that $D_{i}^{(n)}$ sets the ith boundary condition. Using this notation, and taking the cantilever beam problem as example, general boundary conditions for the problem can be written as

$$
\begin{aligned}
& D_{i}^{(n)}[v(0, t)]=f_{i}(t), \quad i=1,2 \\
& D_{i}^{(n)}[v(1, t)]=f_{i}(t), \quad i=3,4
\end{aligned}
$$


where the $f_{i}(t)$ functions depend on the characteristics of each system. It is important to note that for the clamped rod problem, it is necessary to impose only two boundary conditions.

The initial conditions of movement are specified by two arbitrary functions

$$
\begin{gathered}
v(x, 0)=v_{0}(x) \\
\frac{\partial v}{\partial t}(x, 0)=\dot{v}_{0}(x)
\end{gathered}
$$

A difficulty in solving problems of this type by the method of separation of variables arises when not all of the functions $f_{i}(t)$ that define boundary conditions vanish. ${ }^{5}$

In this case, the method of separation of variables breaks down when applied directly because it is impossible to satisfy the boundary conditions define by equations (39(a)) and (39(b)), since the function dependent on $x$ would not be consistent.

The above difficulty can be overcome by separating the solution into two parts, one of which will later be adjusted to simplify the boundary conditions on the other.

Therefore, it will require the displacements that are to be obtained to take the form ${ }^{5}$

$$
v(x, t)=w(x, t)+\sum_{i=1}^{4} f_{i}(t) g_{i}(x)
$$

where $f_{i}(t)$ are the functions shown in equation (39(a)) and (39(b)), which determine the boundary conditions.

When this expression is substituted in the movement equation of the problem, e.g. equation (7), it is found that the new function $w(x, t)$ must satisfy the differential equation

$$
\frac{\partial^{2} w}{\partial t^{2}}+\frac{\partial^{4} w}{\partial x^{4}}+\sum_{i=1}^{4}\left(\ddot{f}_{i} g_{i}+f_{i} g_{i}^{I V}\right)=F(x, t)
$$

Moreover, the expression assumed for $v(x, t)$, equation (41), must satisfy boundary conditions, equation (39(a)) and (39(b)), therefore

$$
\begin{aligned}
& D_{i}^{(n)}[w(0, t)]=f_{i}(t)-\sum_{j=1}^{4} f_{j}(t) D_{i}^{(n)}\left[g_{j}(0)\right], \quad i=1,2 \\
& D_{i}^{(n)}[w(1, t)]=f_{i}(t)-\sum_{j=1}^{4} f_{j}(t) D_{i}^{(n)}\left[g_{j}(1)\right], \quad i=3,4
\end{aligned}
$$

Finally, initial conditions, equations (40(a)) and (40(b)), become

$$
\begin{aligned}
& w(x, 0)=v_{0}(x)-\sum_{i=1}^{4} f_{i}(0) g_{i}(x) \\
& \dot{w}(x, 0)=\dot{v}_{0}(x)-\sum_{i=1}^{4} \dot{f}_{i}(0) g_{i}(x)
\end{aligned}
$$

The next step is to determine the functions $g_{i}(x)$ so that the terms on the right side of equation (43(a)) and (43(b)) are cancelled, giving homogeneous boundary conditions for the function $w(x, t)$. To ensure this, it is sufficient to meet the following conditions

$$
\begin{aligned}
& D_{j}^{(n)}\left[g_{i}(0)\right]=\delta_{i j}, \quad j=1,2, \quad i=1,2,3,4 \\
& D_{j}^{(n)}\left[g_{i}(1)\right]=\delta_{i j}, \quad j=3,4, \quad i=1,2,3,4
\end{aligned}
$$

Each of the expressions given by equation (45(a)) and $(45(b))$, provides four conditions for each of the four functions $g_{i}(x)$. To ensure that these conditions are satisfied in all cases, the functions $g_{i}(x)$ are taken as fifth-degree polynomials in $x$

$$
g_{i}(x)=a_{i}+b_{i} x+c_{i} x^{2}+d_{i} x^{3}+e_{i} x^{4}+h_{i} x^{5}
$$

choosing the coefficients according to the following criterion $^{5}$

Substitute each of the $g_{i}(x)$ in the appropriate expression of equation (45(a)) and (45(b)). Each case will result in a set of four linear algebraic equations governing the coefficients of the particular function $g_{i}(x)$. If more than four of the constants $a_{i} \ldots h_{i}$ appear in these expressions, this constants must be reduced to four by setting equal to zero the coefficient of the term of highest degree in $x$ and also, if necessary, the coefficient of the term of the second highest degree. If any of the constants $a_{i} \ldots h_{i}$ does not appear, they must be set equal to zero. Moreover, it is necessary to calculate only those $g_{i}(x)$ functions for which the corresponding $f_{i}(t)$ are not zero.

The resulting equations determine the constants in equation (46). With this choice of the functions $g_{i}(x)$, the boundary conditions expressed by equation (43(a)) and (43(b)) become those of a stationary problem

$$
\begin{aligned}
& D_{i}^{(n)}[w(0, t)]=0 \quad i=1,2 \\
& D_{i}^{(n)}[w(1, t)]=0 \quad i=3,4
\end{aligned}
$$


It is important to note that for the clamped rod problem, the conditions given by equation (45(a)) and (45(b)) will be reduced to four, and therefore, the fifthdegree polynomials that define the $g_{i}(x)$ functions, reduced to third-degree polynomials.

The last step is to find the function $w(x, t)$ that satisfies the differential equation given by equation (42), with the boundary conditions expressed by equation (47(a)) and (47(b)), and initial conditions given by equation (44(a)) and (44(b)). This is a typical problem of forced vibrations with homogeneous boundary conditions, which can now be solved by the method of separation of variables, looking for a solution that follows the expression

$$
w(x, t)=\sum_{j=1}^{\infty} \phi_{j}(x) \eta_{j}(t)
$$

This completes the formal solution of the problem.

\section{Clamped rod problem}

Returning to the specific clamped rod problem of study, the boundary conditions given by equation (39(a)) and (39(b)) are reduced to two, and taking into account equation (31), can be rewritten as

$$
D_{1}^{(n)}[u(0, t)]=f_{1}(t), \quad D_{2}^{(n)}[u(1, t)]=f_{2}(t)
$$

where

$$
D_{1}^{(n)}=D_{1}^{(0)}, \quad D_{2}^{(n)}=D_{2}^{(1)}
$$

and

$$
f_{1}(t)=0, \quad f_{2}(t)=H P(t)
$$

and, as discussed above, $f_{2}(t)$ being the only non-zero function, it is necessary only to calculate the function $g_{2}(x)$. For this, it will follow the procedure set by the previous criterion, finally obtaining the expression

$$
g_{2}(x)=x
$$

Since $f_{1}(t)=0$, it follows that

$$
u(x, t)=w(x, t)+f_{2}(t) g_{2}(x)
$$

Substituting the expression above into equation (4), adapted to a free-vibration problem, and taking into account, from equation (52), that $g_{2}^{\prime \prime}(x)=0$, the partial differential equation that must satisfy the function $w(x, t)$ is finally

$$
\frac{\partial^{2} w}{\partial t^{2}}-\frac{\partial^{2} w}{\partial x^{2}}=-\ddot{f}_{2}(t) g_{2}(x)=-\ddot{f}_{2}(t) x
$$

With the expression given by equation (52) for $g_{2}(x)$, the boundary conditions for $w(x, t)$ become homogeneous

$$
w(0, t)=w^{\prime}(1, t)=0
$$

and initial conditions become

$$
\begin{aligned}
& w(x, 0)=-f_{2}(0) g_{2}(x)=-f_{2}(0) x, \\
& \dot{w}(x, 0)=-\dot{f}_{2}(0) g_{2}(x)=-\dot{f}_{2}(0) x
\end{aligned}
$$

Equation (54) and (55) correspond to a forcedvibration problem with homogeneous boundary conditions, which will be solved by the method of separation of variables. Thus, the function $w(x, t)$ will be given as

$$
w(x, t)=\sum_{j=1}^{\infty} \phi_{j}(x) \eta_{j}(t)
$$

where functions $\phi_{j}(x)$ are given again by equation (14) and

$$
\begin{aligned}
\eta_{j}(t)= & C_{1 j} \sin \left(\omega_{j} t\right)+C_{2 j} \cos \left(\omega_{j} t\right) \\
& +\frac{G_{2 j}}{\omega_{j}} \int_{0}^{t}\left(-\ddot{f}_{2}(\tau)\right) \sin \left(\omega_{j}(t-\tau)\right) \mathrm{d} \tau
\end{aligned}
$$

with $\omega_{j}$ given by equation (16) and

$$
\begin{gathered}
G_{2 j}=\frac{\int_{0}^{1} g_{2}(x) \phi_{j}(x) \mathrm{d} x}{\int_{0}^{1} \phi_{j}^{2}(x) \mathrm{d} x}=2 \int_{0}^{1} g_{2}(x) \phi_{j}(x) \mathrm{d} x, \\
C_{1 j}=\frac{-\dot{f}_{2}(0) G_{2 j}}{\omega_{j}}, C_{2 j}=-f_{2}(0) G_{2 j}
\end{gathered}
$$

Therefore, the final solution for the displacements of a clamped rod under the action of an external point load at its free end, this being applied as a time-dependent boundary condition, is

$$
\bar{u}(x, t)=u(x, t) L=L\left[\sum_{j=1}^{\infty} \phi_{j}(x) \eta_{j}(t)+H P(t) x\right]
$$

where the functions $\phi_{j}(x)$ and $\eta_{j}(t)$ are given, respectively, by equations (14) and (58).

The axial forces along the rod are again determined by the expression given by equation (18).

\section{Cantilever beam problem}

From equations (36(a) and 36(b)) and (39(a) and 39(b)), we get that

$$
f_{1}(t)=f_{2}(t)=f_{3}(t)=0, \quad f_{4}(t)=K P(t)
$$


and

$$
\begin{aligned}
& D_{1}^{(n)}=D_{1}^{(0)}, \quad D_{2}^{(n)}=D_{2}^{(1)}, \quad D_{3}^{(n)}=D_{3}^{(2)}, \\
& D_{4}^{(n)}=D_{4}^{(3)}
\end{aligned}
$$

Again, being $f_{4}(t)$ the only non-zero function, it is only necessary to calculate the function $g_{4}(x)$. From equation (45(a) and 45(b)), and following the previous criterion, we obtain that

$$
g_{4}(x)=\frac{x^{3}}{6}-\frac{x^{2}}{2}
$$

The transversal displacements of the beam can be now expressed as

$$
v(x, t)=w(x, t)+f_{4}(t) g_{4}(x)
$$

Substituting equation (64) into equation (35), and considering, from equation (63), that $g_{4}{ }^{I V}(x)=0$, the partial differential equation that must satisfy the function $w(x, t)$ for this problem is finally

$$
\frac{\partial^{2} w}{\partial t^{2}}+\frac{\partial^{4} w}{\partial x^{4}}=-\ddot{f}_{4}(t) g_{4}(x)
$$

subjected to the boundary conditions

$$
w(0, t)=w^{\prime}(0, t)=w^{\prime \prime}(1, t)=w^{\prime \prime \prime}(1, t)=0
$$

and initial conditions

$$
w(x, 0)=-f_{4}(0) g_{4}(x), \quad \dot{w}(x, 0)=-\dot{f}_{4}(0) g_{4}(x)
$$

Thus, applying the method of separation of variables, the function $w(x, t)$ will be given again by equation (57), where, for this problem, functions $\phi_{j}(x)$ are given by equation (23) and

$$
\begin{aligned}
\eta_{j}(t)= & C_{1 j} \sin \left(\omega_{j} t\right)+C_{2 j} \cos \left(\omega_{j} t\right) \\
& +\frac{G_{4 j}}{\omega_{j}} \int_{0}^{t}\left(-\ddot{f}_{4}(\tau)\right) \sin \left(\omega_{j}(t-\tau)\right) \mathrm{d} \tau
\end{aligned}
$$

where $\omega_{j}$ is obtained from equation (26) and

$$
\begin{aligned}
G_{4 j} & =\frac{\int_{0}^{1} g_{4}(x) \phi_{j}(x) \mathrm{d} x}{\int_{0}^{1} \phi_{j}^{2}(x) \mathrm{d} x}, \quad C_{1 j}=\frac{-\dot{f}_{4}(0) G_{4 j}}{\omega_{j}}, \\
C_{2 j} & =-f_{4}(0) G_{4 j}
\end{aligned}
$$

Therefore, by this method, the final solution for the displacements of the cantilever beam problem is

$$
\bar{v}(x, t)=v(x, t) L=L\left[\sum_{j=1}^{\infty} \phi_{j}(x) \eta_{j}(t)+K P(t) g_{4}(x)\right]
$$

where the functions $\phi_{j}(x)$ and $\eta_{j}(t)$ are given, respectively, by equations (23) and (68).

The shear force along the beam is determined by the expression given in equation (29(b)).

\section{Comparison of results: practical examples}

So far, a generic applied load has been taken into account. The next step is to particularize the solutions achieved by both methods for a specific load. In this study, a parabolic load given by the following expression

$$
\bar{P}(\bar{t})=-\bar{S} \bar{t}^{2}+2 \bar{T} \bar{t}, \quad 0 \leqslant \bar{t} \leqslant 2
$$

has been considered.

From here on, superscript $(h)$ will be used for functions related to the classical method of resolution, by which homogeneous boundary conditions were considered, and the superscript $(\tau)$ for those related to the method by which boundary conditions were considered time dependent.

\section{Clamped rod problem}

The load given by equation (71) can be expressed in a dimensionless form as

$$
P(t)=-S_{r} t^{2}+2 T_{r} t, \quad 0 \leqslant t \leqslant \frac{2 c_{r}}{L}
$$

For the classical method of resolution $P(t) \equiv P^{h}(t)$, and using equation (5)

$$
S_{r}=S_{r}^{h}=\frac{\bar{S}_{r}^{h} L^{3}}{E A c_{r}^{2}}, \quad T_{r}=T_{r}^{h}=\frac{\bar{T}_{r}^{h} L^{2}}{E A c_{r}}
$$

On the other hand, for the method by which boundary conditions were considered time dependent $P(t) \equiv P^{\tau}(t)$, and using equation (34)

$$
S_{r}=S_{r}^{\tau}=\frac{\bar{S}_{r}^{\tau} L^{2}}{P_{0} c_{r}^{2}}, \quad T_{r}=T_{r}^{\tau}=\frac{\bar{T}_{r}^{\tau} L}{P_{0} c_{r}}
$$

which, using equation (33) and the relations

$$
\bar{S}_{r}^{\tau}=\bar{S}_{r}^{h} L, \quad \bar{T}_{r}^{\tau}=\bar{T}_{r}^{h} L
$$


can be rewritten as

$$
S_{r}^{\tau}=\frac{\bar{S}_{r}^{h} L^{3}}{H E A c_{r}^{2}}, \quad T_{r}^{\tau}=\frac{\bar{T}_{r}^{h} L^{2}}{H E A c_{r}}
$$

The comparison will be made by contrasting the longitudinal displacements of the free end of the rod and the axial force to which the rod is subjected.

Longitudinal displacements. The expression formulated for the longitudinal displacements of the rod, using the classical method of resolution was given by equation (13), where the functions $\phi_{j}(x)$ and $\eta_{j}^{h}(t)$ are given, respectively, by equations (14) and (15).

Specializing these equations for a parabolic $P(t)$ load given by equations (72) and (73), it follows that

$$
\bar{u}^{h}(x, t)=u^{h}(x, t) L=L\left[\sum_{j=1}^{\infty} \phi_{j}(x) \eta_{j}^{h}(t)\right]
$$

and

$$
\begin{aligned}
\eta_{j}^{h}(t)= & \frac{-2 \phi_{j}(1)}{\omega_{j}^{4}}\left[2 T_{r}^{h} \omega_{j} \sin \left(\omega_{j} t\right)+2 S_{r}^{h} \cos \left(\omega_{j} t\right)\right. \\
& \left.+\omega_{j}^{2} t\left(S_{r}^{h} t-2 T_{r}^{h}\right)-2 S_{r}^{h}\right]
\end{aligned}
$$

On the other hand, the expression formulated for the longitudinal displacements of the rod, using the timedependent boundary conditions method of resolution, were given by equation (60), where the functions $\phi_{j}(x)$ and $\eta_{j}^{\tau}(t)$ are given, respectively, by equations (14) and (58).

Again, specializing the equations (58) and (60) for a parabolic $P(t)$ load given by equations (72) and (76), it follows that

$$
\begin{aligned}
\bar{u}^{\tau}(x, t) & =u^{\tau}(x, t) L \\
& =L\left[\sum_{j=1}^{\infty} \phi_{j}(x) \eta_{j}^{\tau}(t)+H\left(-S_{r}^{\tau} t^{2}+2 T_{r}^{\tau} t\right) x\right]
\end{aligned}
$$

and

$$
\eta_{j}^{\tau}(t)=\frac{-2 H G_{2 j}}{\omega_{j}^{2}}\left[S_{r}^{\tau} \cos \left(\omega_{j} t\right)+T_{r}^{\tau} \omega_{j} \sin \left(\omega_{j} t\right)-S_{r}^{\tau}\right]
$$

Thus, the full expressions for the displacements, referring to the free end of the rod, $x=1$, are

$$
\begin{aligned}
u^{h}(1, t)= & \sum_{j=1}^{\infty} \frac{-4}{\omega_{j}^{4}}\left[S_{r}^{h} \cos \left(\omega_{j} t\right)+T_{r}^{h} \omega_{j} \sin \left(\omega_{j} t\right)-S_{r}^{h}\right] \\
& +\sum_{j=1}^{\infty} \frac{-4 t}{\omega_{j}^{2}}\left(\frac{S_{r}^{h} t}{2}-T_{r}^{h}\right)
\end{aligned}
$$

$$
\begin{aligned}
u^{\tau}(1, t)= & \sum_{j=1}^{\infty} \frac{-4}{\omega_{j}^{4}}\left[S_{r}^{h} \cos \left(\omega_{j} t\right)+T_{r}^{h} \omega_{j} \sin \left(\omega_{j} t\right)-S_{r}^{h}\right] \\
& +\left(2 T_{r}^{h} t-S_{r}^{h} t^{2}\right)
\end{aligned}
$$

In formulating the equation (81(a)) and (81(b)), the following relations have been taken into account

$$
\begin{gathered}
G_{2 j} \phi_{j}(1)=2 \phi_{j}(1) \int_{0}^{1} g_{2}(x) \phi_{j}(x) \mathrm{d} x=\frac{2}{\omega_{j}^{2}}, \\
S_{r}^{h}=S_{r}^{\tau} H, \quad T_{r}^{h}=T_{r}^{\tau} H
\end{gathered}
$$

Note that the first term of equation (81(a)) and (81(b)) is null for all values of $j$, so the displacements $u^{h}(1, t)$ and $u^{\tau}(1, t)$ can be expressed as

$$
\begin{aligned}
& u^{h}(1, t)=\sum_{j=1}^{\infty} \frac{-4 t}{\omega_{j}^{2}}\left(\frac{S_{r}^{h} t}{2}-T_{r}^{h}\right) \\
& u^{\tau}(1, t)=\left(2 T_{r}^{h} t-S_{r}^{h} t^{2}\right)
\end{aligned}
$$

In view of equation (83(a)) and (83(b)), it follows that to ensure the equality in displacement, that is

$$
\frac{u^{h}(1, t)}{u^{\tau}(1, t)}=1
$$

it must be satisfied that

$$
\sum_{j=1}^{\infty} \frac{-4 t}{\omega_{j}^{2}}\left(\frac{S_{r}^{h} t}{2}-T_{r}^{h}\right)=2 T_{r}^{h} t-S_{r}^{h} t^{2}
$$

This leads to the following two equalities

$$
\begin{aligned}
& \sum_{j=1}^{\infty} \frac{-4 S_{r}^{h}}{2 \omega_{j}^{2}}=-S_{r}^{h} \\
& \sum_{j=1}^{\infty} \frac{4 T_{r}^{h}}{\omega_{j}^{2}}=2 T_{r}^{h}
\end{aligned}
$$

As can be seen, the last two expressions lead to the same condition, given as

$$
\sum_{j=1}^{\infty} \frac{2}{\omega_{j}^{2}}=1
$$

which have no dependence on the type of load applied, and depend only on the value of the natural frequencies of vibration $\omega_{j}$.

Defining the parameter $R_{1}(n)$ as

$$
R_{1}(n)=\sum_{j=1}^{n} \frac{2}{\omega_{j}^{2}}
$$


can be observed from equation (87), that equation (84) is fulfilled when

$$
R_{1 \infty}=\sum_{j=1}^{\infty} \frac{2}{\omega_{j}^{2}}=1
$$

To verify whether the above equality holds, Figure 3 graphically shows the values taken by the parameter $R_{1}(n)$ as $n$ increase to a maximum of $n=20$.

As shown in Figure 3, as more terms are being taken in the series, the result converges to 1 . If only the first mode of vibration is taken into account to compute the solution achieved using the classical method, that is, if only the first term of the series is considered, the difference between the solution of the two methods is approximately $19 \%$. As shown in the graph, however, if to reach the solution by the classical method, the first six modes are taken into account, this difference is reduced to approximately $3.37 \%$.

Therefore, it has been shown that the displacements achieved by both methods are exactly the same for an infinite number of vibration modes, since the infinite series given by equation (89) converges to 1 . However, it is important to note the large existing difference, almost $20 \%$, if only the first mode of vibration is considered in the calculation of the solution by the classical method.

In addition, from this result, an important conclusion can be achieved. If taking into account a considerable number of vibration modes to calculate the longitudinal displacements, the two methods lead to the same result regardless of the type of load being applied to the system. This can be seen on the condition given by equation (89), which have no dependence on the type of load applied and depend only on the value of the natural frequencies of vibration $\omega_{j}$.

Axial force. The next step is to perform the same comparative study, but this time with the axial force at the free end of the rod, $N(1, t)$. Again, the subscripts $(h)$ and $(\tau)$ will be used to refer to the axial forces made by each method.

The expression that relates the axial force with the longitudinal displacements of the rod is given by equation (18). Thus, from equation (77), the axial force on the rod, according to the classical method of resolution, will be defined by

$$
N^{h}(x, t)=\sum_{j=1}^{\infty} \phi_{j}^{\prime}(x) \eta_{j}^{h}(t)
$$

where the vibration modes, $\phi_{j}(x)$, are again of the form given by equation (14), and the functions $\eta_{j}^{h}(t)$ are given by equation (78). Also, from equation (79), the axial force on the rod, according to the time-dependent boundary conditions method of resolution, will be defined as

$$
N^{\tau}(x, t)=\sum_{j=1}^{\infty} \phi_{j}^{\prime}(x) \eta_{j}^{\tau}(t)+H\left(-S_{r}^{\tau} t^{2}+2 T_{r}^{\tau} t\right)
$$

where the functions $\eta_{j}^{\tau}(t)$ are given by equation (80).

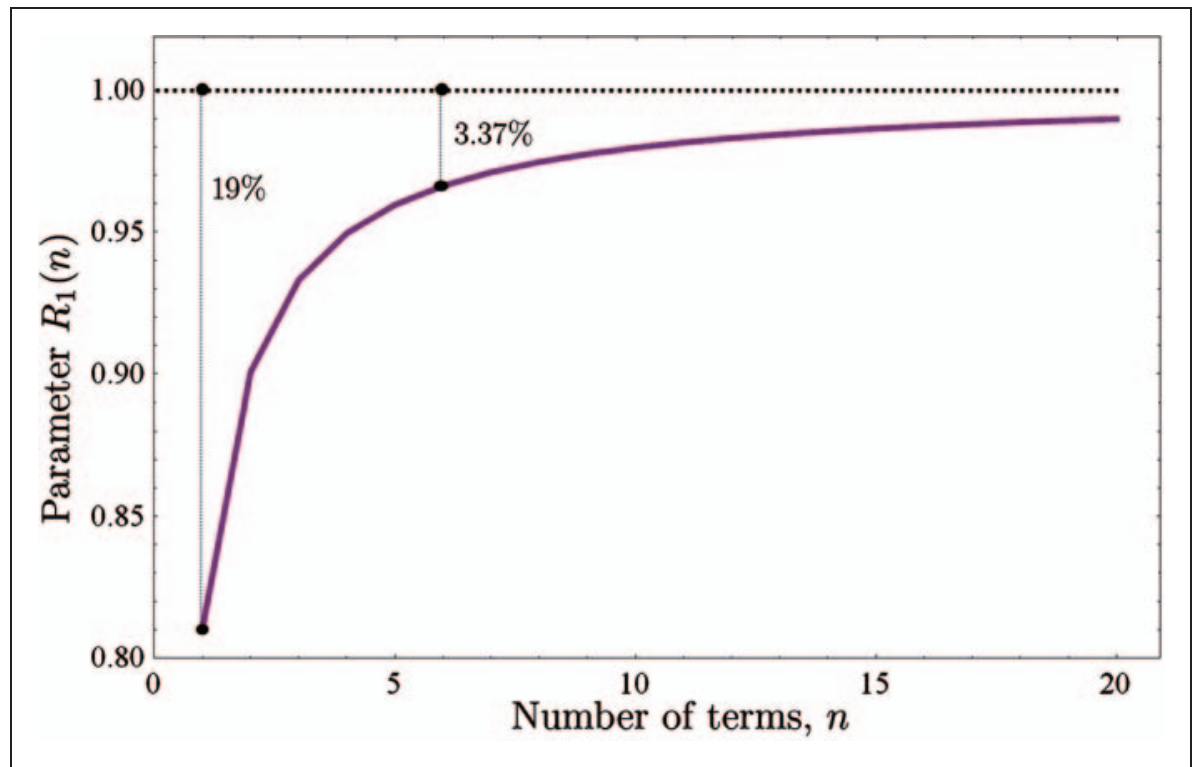

Figure 3. Ratio between longitudinal displacements of the free end of the rod obtained by both methods versus the number of eigenmodes considered. 
Therefore, the axial force at the free end of the rod for each of the two resolution methods is given by

$$
\begin{gathered}
N^{h}(1, t)=\sum_{j=1}^{\infty} \frac{-4 \phi_{j}^{\prime}(1) \phi_{j}(1)}{\omega_{j}^{4}}\left[S_{r}^{h} \cos \left(\omega_{j} t\right)\right. \\
\left.+T_{r}^{h} \omega_{j} \sin \left(\omega_{j} t\right)-S_{r}^{h}+\omega_{j}^{2} t\left(\frac{S_{r}^{h} t}{2}-T_{r}^{h}\right)\right] \\
N^{\tau}(1, t)=\sum_{j=1}^{\infty} \frac{-2 \phi_{j}^{\prime}(1) H G_{2 j}}{\omega_{j}^{2}}\left[S_{r}^{\tau} \cos \left(\omega_{j} t\right)\right. \\
\left.+T_{r}^{\tau} \omega_{j} \sin \left(\omega_{j} t\right)-S_{r}^{\tau}\right]+H\left(-S_{r}^{\tau} t^{2}+2 T_{r}^{\tau} t\right)
\end{gathered}
$$

It is important to note that in both expressions the term $\phi_{j}^{\prime}(1)$ appears, which determine the boundary condition that was applied at the free end of the rod when calculating the displacement $u^{h}(x, t)$ and the $w(x, t)$ function. In both cases, the boundary conditions were homogeneous, so that

$$
\phi_{j}^{\prime}(1)=0
$$

Therefore, it is easy to see, using equation (93), that both axial forces at the free end of the rod are different, since

$$
\begin{aligned}
& N^{h}(1, t)=0 \\
& N^{\tau}(1, t)=H\left(-S_{r}^{\tau} t^{2}+2 T_{r}^{\tau} t\right)
\end{aligned}
$$

This implies that, as was demonstrated, although the longitudinal displacements achieved by both methods were equal at the infinite limit, the results achieved for the axial force at the free end of the rod were not equal, being calculated by both methods.
It can be noted, in view of equations (51), (72) and (94(b)) that, according to the time-dependent boundary-conditions method of resolution, the axial force at the free end of the rod coincides at all times with the one that would be produced by an axial force.

Figure 4 shows the axial forces along the rod for a fixed time, in this case $t=c_{r} / L$. This graph highlights the differences between the results given by both methods.

It can be noted that while the axial force obtained with time-dependent boundary conditions, $N^{\tau}$, is constant along the rod, the one obtained by the classic variable-separation method, that is, $N^{h}$, is not a constant value. Therefore, it is shown that the axial force $N^{h}$ is null on the free end of the rod.

\section{Cantilever beam problem}

For this problem, the dimensionless form of the load given by equation (71), can be expressed as

$$
P(t)=-S_{b} t^{2}+2 T_{b} t, \quad 0 \leqslant t \leqslant \frac{2 c_{b}}{L^{2}}
$$

Following the same methodology as for the previous case, we have that

$$
\begin{gathered}
S_{b}^{h}=\frac{\bar{S}_{b}^{h} L^{7}}{E I c_{b}^{2}}, \quad T_{b}^{h}=\frac{\bar{T}_{b}^{h} L^{5}}{E I c_{b}} \\
S_{b}^{\tau}=\frac{\bar{S}_{b}^{h} L^{7}}{K E I c_{b}^{2}}, \quad T_{b}^{\tau}=\frac{\bar{T}_{b}^{h} L^{5}}{K E I c_{b}}
\end{gathered}
$$

In this case, the comparison will be made between the transversal displacements of the free end of the beam, and the shear force to which the beam is subjected.

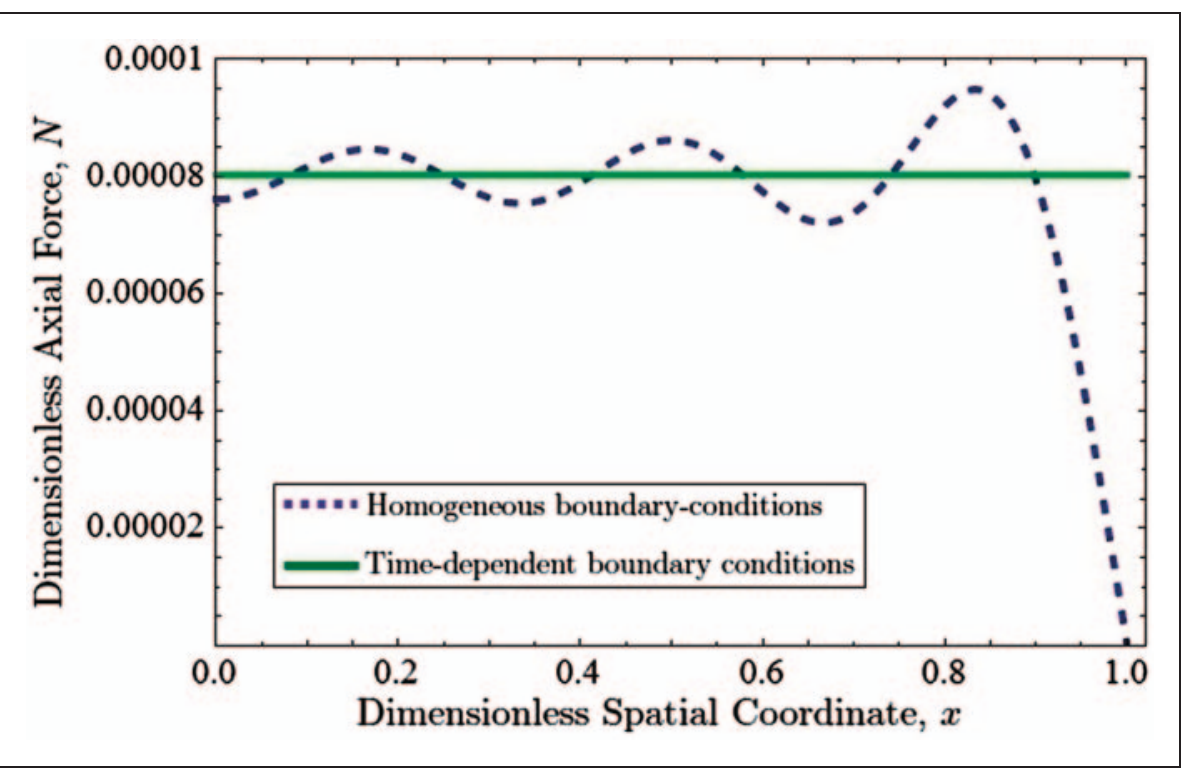

Figure 4. Dimensionless axial force along the rod for $t=c_{r} / L$. 
Transversal displacements. From previous sections and following the same steps as for the clamped rod problem, we have that the transversal displacements at the free end of the beam, obtained by the two methods, are

$$
\begin{aligned}
& v^{h}(1, t) \\
& =\sum_{j=1}^{\infty} \frac{-2 \phi_{j}^{2}(1)}{\omega_{j}^{3} \int_{0}^{1} \phi_{j}^{2}(x) \mathrm{d} x} \times\left\{\frac{S_{b}^{h}}{\omega_{j}}\left[1-\cos \left(\omega_{j} t\right)\right]-T_{b}^{h} \sin \left(\omega_{j} t\right)\right\} \\
& +\sum_{j=1}^{\infty} \frac{-\phi_{j}^{2}(1)}{\omega_{j}^{2} \int_{0}^{1} \phi_{j}^{2}(x) \mathrm{d} x}\left(-S_{b}^{h} t^{2}+2 T_{b}^{h} t\right) \\
& v^{\tau}(1, t) \\
& =\sum_{j=1}^{\infty} \frac{2 \phi_{j}(1) G_{4 j}}{\omega_{j}}\left\{\frac{S_{b}^{h}}{\omega_{j}}\left[1-\cos \left(\omega_{j} t\right)\right]-T_{b}^{h} \sin \left(\omega_{j} t\right)\right\} \\
& +g_{4}(1)\left(-S_{b}^{h} t^{2}+2 T_{b}^{h} t\right)
\end{aligned}
$$

In this case, the first terms of both expressions are not zero, as in the bar problem, but can be verified that these terms are three to four orders of magnitude smaller than the second terms on both equations. For that reason, the displacements given by equation (98(a)) and (98(b)) can be approximated by

$$
\begin{aligned}
& v^{h}(1, t) \approx \sum_{j=1}^{\infty} \frac{-\phi_{j}^{2}(1)}{\omega_{j}^{2} \int_{0}^{1} \phi_{j}^{2}(x) \mathrm{d} x}\left(-S_{b}^{h} t^{2}+2 T_{b}^{h} t\right) \\
& v^{\tau}(1, t) \approx g_{4}(1)\left(-S_{b}^{h} t^{2}+2 T_{b}^{h} t\right)
\end{aligned}
$$

As in the previous case, to ensure the equality between displacements, it must be satisfied the following equality

$$
\sum_{j=1}^{\infty} \frac{-\phi_{j}^{2}(1)}{\omega_{j}^{2} \int_{0}^{1} \phi_{j}^{2}(x) \mathrm{d} x}=g_{4}(1)=-\frac{1}{3}
$$

To check if previous condition is met and observe the differences between the results provided by both methods depending on the number of terms considered in the series, the parameter $R_{2}(n)$ will be defined as

$$
R_{2}(n)=\sum_{j=1}^{n} \frac{3 \phi_{j}^{2}(1)}{\omega_{j}^{2} \int_{0}^{1} \phi_{j}^{2}(x) \mathrm{d} x}
$$

It can be seen that transverse displacements calculated by both methods are equal when

$$
R_{2 \infty}=\sum_{j=1}^{\infty} \frac{3 \phi_{j}^{2}(1)}{\omega_{j}^{2} \int_{0}^{1} \phi_{j}^{2}(x) \mathrm{d} x}=1
$$

Figure 5 graphically shows the values taken by the parameter $R_{2}(n)$ as $n$ increase from 1 to 20 .

In Figure 5, it does appear a clear relationship between the number of terms considered in the series given by equation (101), and the differences between the solutions provided by both methods.

In view of this, and just as in the clamped rod problem, it can be seen that the transversal displacements derived by both methods are the same for an infinite number of vibrations modes, since the series given by equation (101) converges to 1 as $n \rightarrow \infty$.

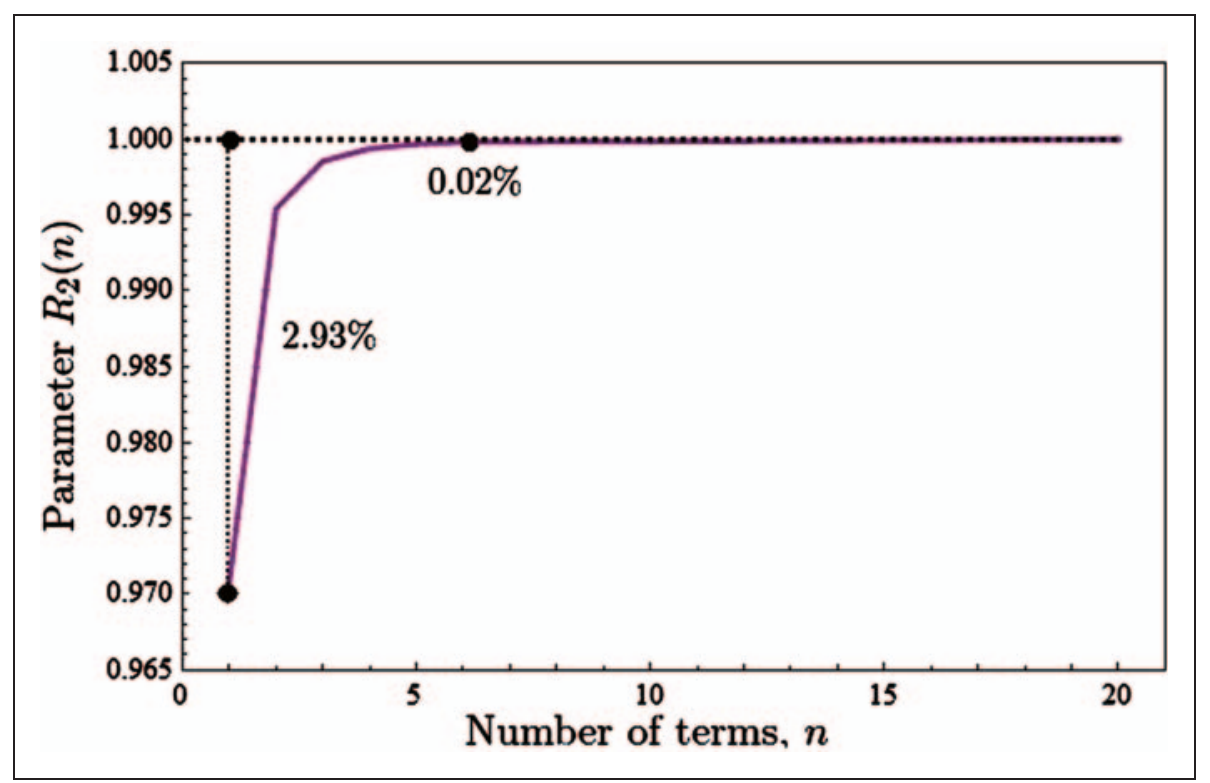

Figure 5. Ratio between transversal displacements of the free end of the beam obtained by both methods versus the number of eigenmodes considered. 
It can also be seen in Figure 5 that if only one vibration mode, i.e. if only one term of the solution given by equation (22) is considered, the difference between the solutions provided by both methods increase to $3 \%$. This difference is reduced to $0.02 \%$ when the first six eigenmodes are used.

Shear Force. From the equations (22), (29(b)), and (70), the shear force at the free end of the beam can be obtained using the two methods of solution introduced. These forces are given by

$$
\begin{aligned}
V^{h}(1, t)= & \sum_{j=1}^{\infty} \frac{2 \phi_{j}^{\prime \prime \prime}(1) \phi_{j}(1)}{\omega_{j}^{3} \int_{0}^{1} \phi_{j}^{2}(x) \mathrm{d} x} \\
& \times\left\{\frac{S_{b}^{h}}{\omega_{j}}\left[1-\cos \left(\omega_{j} t\right)\right]-T_{b}^{h} \sin \left(\omega_{j} t\right)\right\} \\
& +\sum_{j=1}^{\infty} \frac{\phi_{j}^{\prime \prime \prime}(1) \phi_{j}(1)}{\omega_{j}^{2} \int_{0}^{1} \phi_{j}^{2}(x) \mathrm{d} x}\left(-S_{b}^{h} t^{2}+2 T_{b}^{h} t\right) \\
V^{\tau}(1, t)= & \sum_{j=1}^{\infty} \frac{-2 \phi_{j}^{\prime \prime \prime}(1) K G_{4 j}}{\omega_{j}} \\
& \times\left\{\frac{A_{b}^{\tau}}{\omega_{j}}\left[1-\cos \left(\omega_{j} t\right)\right]-B_{b}^{\tau} \sin \left(\omega_{j} t\right)\right\} \\
& -K\left(-A_{b}^{\tau} t^{2}+2 B_{b}^{\tau} t\right)
\end{aligned}
$$

Exactly as in the clamped rod problem, in equation (103(a)) and (103(b)) appears the term $\phi_{j}^{\prime \prime \prime}(1)$. This determines the boundary condition that was applied at the free end of the beam when the transversal displacement $v^{h}(x, t)$ and the $w(x, t)$ function were calculated. In both cases, it was hold that

$$
\phi_{j}^{\prime \prime \prime}(1)=0
$$

Therefore, the shear forces at the free end of the beam differ according to the method used

$$
\begin{aligned}
& V^{h}(1, t)=0 \\
& V^{\tau}(1, t)=K\left(-S_{b}^{\tau} t^{2}+2 T_{b}^{\tau} t\right)
\end{aligned}
$$

Moreover, as with the axial force on the rod problem, Figure 6 shows as constant the shear force obtained with time-dependent boundary conditions, $V^{\tau}$, but that the value calculated by the classic variable separation method, $V^{h}$ is not a constant one.

\section{Concluding remarks}

In this article, the axial vibrations of a rod with a clamped end and the transversal vibrations of a cantilever beam, both with a time-dependent and non-harmonic force $P(t)$ applied on its free end, are analysed. The solutions are achieved using the method proposed by Mindlin and Goodman ${ }^{5}$ and the results are compared with those calculated by the classic variableseparation method (which does not properly accomplish the time-dependent boundary conditions). Regarding the displacement of the free end, both solutions converge if a sufficient number of vibration modes are used. However, in the clamped rod problem, if only the fundamental mode is retained, a difference close to $19 \%$ arises, and a reduction of this difference to $1 \%$

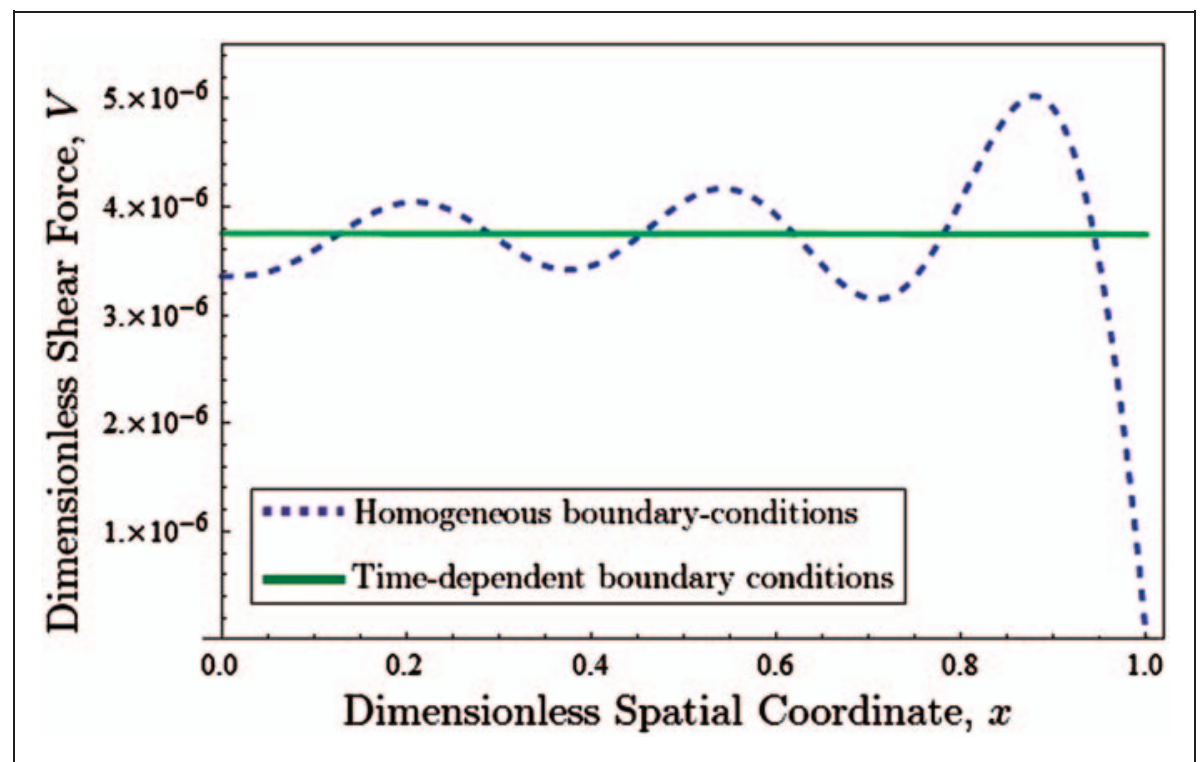

Figure 6. Dimensionless shear force along the beam for $t=c_{b} / L$. 
would require the use of the first 20 vibration modes. For the cantilever beam problem, the differences between the solutions provided by the two methods are reduced. In the latter case, a difference close to $3 \%$ arises when only the fundamental mode of vibration is considered, while only a deviation of $0.02 \%$ is obtained when the first six modes are retained.

On the other hand, both methods lead to different axial forces in the rod problem and different shear forces in the beam problem. By the classic variable separation method, is found that these forces are zero at the free ends of both the rod and the beam, which is a logical development of the method, since it imposes zero boundary conditions in terms of forces on the free ends. Otherwise, with the use of the method proposed by Mindlin and Goodman, ${ }^{5}$ the result is more suited to reality since both forces coincide at all times with those applied at the free ends on both problems.

In any case, the differences are independent of the type of time-dependent load exerted at the free end of the rod when this force is non-harmonic. The differences are related to normal modes corresponding to the free vibrations of the mechanical element.

These results highlight the importance of using the proper method of solving the vibration problems with time-dependent boundary conditions.

\section{Funding}

The authors would like to thank the Comisión Interministerial de Ciencia y Tecnología of the Spanish Government and to the Comunidad Autónoma de Madrid for partial support of this work through the research projects DPI2011-23191 and CCG10-UC3M-DPI-5596, respectively.

\section{References}

1. Humar JL. Dynamics of structures, 2nd edn. The Netherlands: A.A. Balkema Publishers, 2002.

2. Benaroya H. Mechanical vibration: analysis, uncertainties, and control. New Jersey: Prentice Hall, 2002.

3. Clough RW and Penzien J. Dynamics of structures, 2nd edn. New York: McGraw-Hill, 1993.

4. Shabana AA. Theory of vibration. New York: Springer, 1991.

5. Mindlin RD and Goodman LE. Beam vibrations with time-dependent boundary conditions. J Appl Mech 1950; 17: 377-380.

6. Courant R and Hilbert D. Methods of mathematical physics. vol. 1, New York: Interscience, 1961, p.277.

\section{Appendix}

Notation

$\begin{array}{ll}A & \text { cross section } \\ c & \text { material-dependent constant }\end{array}$

\begin{tabular}{|c|c|}
\hline$C_{1}, C_{2}$ & $\begin{array}{l}\text { constants determined by initial } \\
\text { conditions }\end{array}$ \\
\hline$D$ & dimensionless constant \\
\hline$D^{(n)}$ & differential operator of order $n$ \\
\hline$E$ & Young's modulus \\
\hline$f$ & function that defines the \\
\hline $\bar{F}, F$ & $\begin{array}{l}\text { time-dependent boundary conditions } \\
\text { dimensional and dimensionless force } \\
\text { applied }\end{array}$ \\
\hline$g$ & spatial dependent auxiliary function \\
\hline$G_{2}, G_{4}, H$ & dimensionless constants \\
\hline$I$ & moment of inertia \\
\hline$K$ & dimensionless constant \\
\hline$L$ & length of the rod or beam \\
\hline $\bar{M}, M$ & $\begin{array}{l}\text { dimensional and dimensionless } \\
\text { bending moment }\end{array}$ \\
\hline $\bar{N}, N$ & $\begin{array}{l}\text { dimensional and dimensionless axial } \\
\text { force }\end{array}$ \\
\hline $\bar{P}(\bar{t}), P(t)$ & $\begin{array}{l}\text { dimensional and dimensionless } \\
\text { time-dependent force }\end{array}$ \\
\hline$P_{0}$ & reference force value \\
\hline$R_{1}(n), R_{2}(n)$ & parameters defined by sums series \\
\hline$R_{1 \infty}, R_{2 \infty}$ & parameters defined by sums series \\
\hline$S, T$ & $\begin{array}{l}\text { dimensionless constants used to define } \\
\text { the parabolic load }\end{array}$ \\
\hline $\bar{S}, \bar{T}$ & $\begin{array}{l}\text { dimensional constants used to define } \\
\text { the parabolic load }\end{array}$ \\
\hline $\bar{t}, t$ & dimensional and dimensionless time \\
\hline $\bar{u}, u$ & $\begin{array}{l}\text { dimensional and dimensionless } \\
\text { longitudinal displacement }\end{array}$ \\
\hline $\bar{v}, v$ & $\begin{array}{l}\text { dimensional and dimensionless } \\
\text { transversal displacement }\end{array}$ \\
\hline $\bar{V}, V$ & $\begin{array}{l}\text { dimensional and dimensionless shear } \\
\text { force }\end{array}$ \\
\hline$w(x, t)$ & auxiliary function \\
\hline $\bar{x}, x$ & $\begin{array}{l}\text { dimensional and dimensionless spatial } \\
\text { coordinate }\end{array}$ \\
\hline$(\cdot)_{b}$ & $\begin{array}{l}\text { subscript relative to the cantilever } \\
\text { beam problem }\end{array}$ \\
\hline$(\cdot)^{h}$ & $\begin{array}{l}\text { superscript relative to the classical } \\
\text { method of resolution }\end{array}$ \\
\hline$(\cdot)_{r}$ & $\begin{array}{l}\text { subscript relative to the clamped rod } \\
\text { problem }\end{array}$ \\
\hline$(\cdot)^{\tau}$ & $\begin{array}{l}\text { superscript relative to the } \\
\text { time-dependent method of resolution }\end{array}$ \\
\hline$\delta(x)$ & Dirac delta function \\
\hline$\delta_{i j}$ & Kronecker delta \\
\hline$\eta$ & modal coordinate \\
\hline$\rho$ & density \\
\hline$\phi$ & modes of vibration \\
\hline & dimensionless vibration frequencies \\
\hline
\end{tabular}

constants determined by initial conditions differential operator of order $n$ Young's modulus the dimensionless constants moment of inertia dimensionless constant ength of the rod or beam dimensional and dimensionless bending momen mensional and dimensionless axial dimensional and dimensionless parameters defined by sums series dimensionless constants used to define the parabolic load the parabolic load dimensional and dimensionless time dimensional and dimensionless ongitudinal displacemen mensional and dimensionless dimensional and dimensionless shear auxiliary function coordinate subscript relative to the cantilever superscript relative to the classical method of resolution subscript relative to the clamped rod problem superscript relative to the

Dirac delta function modes of vibration 